TRANSACTIONS OF THE

AMERICAN MATHEMATICAL SOCIETY

Volume 356, Number 5, Pages 2123-2135

S 0002-9947(04)03468-3

Article electronically published on January 6, 2004

\title{
THE STEEPEST POINT OF THE BOUNDARY LAYERS OF SINGULARLY PERTURBED SEMILINEAR ELLIPTIC PROBLEMS
}

\author{
T. SHIBATA
}

\begin{abstract}
We consider the nonlinear singularly perturbed problem

$$
-\epsilon^{2} \Delta u=f(u), u>0 \quad \text { in } \Omega, u=0 \quad \text { on } \partial \Omega,
$$

where $\Omega \subset \mathbf{R}^{N}(N \geq 2)$ is an appropriately smooth bounded domain and $\epsilon>0$ is a small parameter. It is known that under some conditions on $f$, the solution $u_{\epsilon}$ corresponding to $\epsilon$ develops boundary layers when $\epsilon \rightarrow 0$. We determine the steepest point of the boundary layers on the boundary by establishing an asymptotic formula for the slope of the boundary layers with exact second term.
\end{abstract}

\section{INTRODUCTION}

We consider the following nonlinear singularly perturbed problem:

$$
\begin{aligned}
-\epsilon^{2} \Delta u & =f(u) \text { in } \Omega, \\
u & >0 \text { in } \Omega, \\
u & =0 \text { on } \partial \Omega,
\end{aligned}
$$

where $\Omega \subset \mathbf{R}^{N}(N \geq 2)$ is an appropriately smooth bounded domain satisfying uniform interior and exterior sphere conditions and $\epsilon>0$ is a small parameter. We assume that $f$ satisfies the following:

(f.1) $f \in C^{1, \gamma}(\mathbf{R})$, where $0<\gamma<1$ is a constant.

(f.2) There exists $u_{0}>0$ such that $f(0)=f\left(u_{0}\right)=0$ and $f(u)>0$ for $0<u<u_{0}$.

(f.3) $f^{\prime}(0)>0$ and $f^{\prime}\left(u_{0}\right)<0$.

(f.4) If there exist $u_{1}, u_{2}>0$ such that $u_{0}<u_{1}<u_{2}, f\left(u_{1}\right)=f\left(u_{2}\right)=0, f>0$ in $\left(u_{1}, u_{2}\right)$, then there exists $\tilde{u} \in\left[0, u_{2}\right)$ such that $\int_{\tilde{u}}^{u_{2}} f(s) d s \leq 0$.

The most typical examples of $f$ are $f(u)=u-|u|^{p-1} u(p>1)$ and $f(u)=\sin u$, which are called logistic equation in population dynamics and equation of simple pendulum, respectively, and have been considered by many authors. In these cases, we know that $u_{0}=1$ (resp. $u_{0}=\pi$ ), $u_{\epsilon}<1$ (resp. $u_{\epsilon}<\pi$ ) and $u_{\epsilon} \rightarrow 1$ (resp. $u_{\epsilon} \rightarrow \pi$ ) uniformly on any compact subset in $\Omega$ as $\epsilon \rightarrow 0$. Indeed, under the conditions (f.1)-(f.4), the following facts hold (cf. [3]):

(p.1) For a given $0<\epsilon \ll 1$, there exists a unique solution $u_{\epsilon} \in C^{2}(\bar{\Omega})$ of (1.1)-(1.3).

Received by the editors October 3, 2002 and, in revised form, July 11, 2003.

2000 Mathematics Subject Classification. Primary 35J65, 35J60.

Key words and phrases. Boundary layer, singular perturbation, semilinear elliptic equations.

(C)2004 American Mathematical Society 
(p.2) $\left\|u_{\epsilon}\right\|_{\infty}<u_{0}$.

(p.3) $u_{\epsilon} \rightarrow u_{0}$ uniformly on any compact subset $K \subset \Omega$ as $\epsilon \rightarrow 0$.

Therefore, we see that $u_{\epsilon}$ develops boundary layers as $\epsilon \rightarrow 0$. We also refer to [2][7], 9] and the references therein. Hence, a natural question raised immediately is to ask where on the boundary the steepest point of the boundary layers is situated, and it is the purpose of this paper to answer this question by establishing the two-term asymptotic formula for the slope of the boundary layers as $\epsilon \rightarrow 0$.

Now we state our results. Let $F(u):=\int_{0}^{u} f(s) d s$.

Theorem 1.1. Let $P \in \partial \Omega$ be fixed. Let $\nu$ be a unit outer normal to $\partial \Omega$ at $P$. Then the following asymptotic formula holds as $\epsilon \rightarrow 0$ :

$$
\frac{\partial u_{\epsilon}}{\partial \nu}(P)=-\sqrt{2 F\left(u_{0}\right)} \epsilon^{-1}+\frac{(N-1) C_{0} H(P)}{\sqrt{2 F\left(u_{0}\right)}}+o(1),
$$

where

$$
C_{0}:=\int_{0}^{u_{0}} \sqrt{2\left(F\left(u_{0}\right)-F(s)\right)} d s
$$

and $H(P)$ is the mean curvature of $\partial \Omega$ at $P$.

Therefore, if $P_{1} \in \partial \Omega$ is the only point which attains the minimum of the mean curvature of $\partial \Omega$, then $P_{1}$ is the steepest point of the boundary layers. We remark that the first term in (1.4) has been obtained in [5] when $f(u)=u-|u|^{p-1} u+$ perturbation. When $\Omega=B_{R}$ or $A_{a, R}$, the special cases, (1.4) has been obtained in 11] and [12.

\section{Proof of Theorem 1.1}

We first introduce a diffeomorphism which straightens the boundary portion near a point $P \in \partial \Omega$ (cf. [10]).

Through translation and rotation of the coordinate system, we may assume that $P$ is the origin and the inner normal to $\partial \Omega$ at $P$ is pointing in the direction of the positive $x_{N}$ axis. Let $x^{\prime}=\left(x_{1}, x_{2}, \cdots, x_{N-1}\right)$. Then there exists a smooth function $\psi\left(x^{\prime}\right)$, defined for $\left|x^{\prime}\right| \ll 1$, which satisfies:

$(\psi 1) \psi(0)=0$,

$(\psi 2) \nabla \psi(0)=0$

$(\psi 3) \partial \Omega \cap M=\left\{\left(x^{\prime}, x_{N}\right): x_{N}=\psi\left(x^{\prime}\right)\right\}$,

$(\psi 4) \Omega \cap M=\left\{\left(x^{\prime}, x_{N}\right): x_{N}>\psi\left(x^{\prime}\right)\right\}$,

where $M$ is a neighborhood of $P=0$. For $y \in \mathbf{R}^{N}$ near 0 , we define a mapping $x=\Phi(y)=\left(\Phi_{1}(y), \Phi_{2}(y), \cdots, \Phi_{N}(y)\right)$ by

$$
\begin{aligned}
\Phi_{j}(y) & =y_{j}-y_{N} \frac{\partial \psi}{\partial x_{j}}\left(y^{\prime}\right) \quad(j=1,2, \cdots, N-1), \\
\Phi_{N}(y) & =y_{N}+\psi\left(y^{\prime}\right) .
\end{aligned}
$$

Then $\Phi$ has the inverse mapping $y=\Phi^{-1}(x)$ for $|x|<b$, where $b>0$ is a constant. Let $B_{b / \epsilon}^{+}:=\left\{z \in \mathbf{R}^{N}:|z|<b / \epsilon, z_{N}>0\right\}$. We put

$$
w_{\epsilon}(z):=u_{\epsilon}(\Phi(\epsilon z)), \quad z \in B_{b / \epsilon} .
$$

Then we see from [10, Lemma 4.1] that for $z \in B_{b / \epsilon}^{+}, w_{\epsilon}$ satisfies

$$
\sum_{i, j=1}^{N}\left(\delta_{i j}+2 \epsilon \psi_{i j} z_{N}+\alpha_{i j}(z)\right) \frac{\partial^{2} w_{\epsilon}}{\partial z_{i} \partial z_{j}}+\epsilon \sum_{j=1}^{N} b_{j}(z) \frac{\partial w_{\epsilon}}{\partial z_{j}}+f\left(w_{\epsilon}\right)=0
$$


where

$$
\begin{aligned}
\psi_{i j} & =\frac{\partial^{2} \psi}{\partial x_{i} \partial x_{j}}(0), \\
\psi_{i N} & =\psi_{N j}=0 \text { for } i, j=1, \cdots, N, \\
b_{j}(z) & =-\delta_{j N}(N-1) H(0)+\beta_{j}(z), \\
\left|\alpha_{i j}(z)\right| & \leq C_{2} \epsilon^{2}|z|^{2} \text { for } z \in B_{b / \epsilon}^{+}, \\
\left|\beta_{j}(z)\right| & \leq C_{2} \epsilon|z| \quad \text { for } z \in B_{b / \epsilon}^{+} .
\end{aligned}
$$

To calculate $\left(\partial u_{\epsilon} / \partial \nu\right)(0)$ for $0<\epsilon \ll 1$, we precisely study the asymptotic behavior of $\left(\partial w_{\epsilon} / \partial z_{N}\right)(0)$ as $\epsilon \rightarrow 0$. Let $w$ be a unique solution of

$$
\begin{aligned}
-w^{\prime \prime}(t) & =f(w(t)), \quad t>0, \\
w(0) & =0, \\
w^{\prime}(0) & =\sqrt{2 F\left(u_{0}\right)} .
\end{aligned}
$$

Then we know from $[3]$ that

$$
\begin{aligned}
w^{\prime}(t) & >0, \quad t>0, \\
\lim _{t \rightarrow \infty} w(t) & =u_{0}, \quad \lim _{t \rightarrow \infty} w^{\prime}(t)=0 .
\end{aligned}
$$

Lemma 2.1. Let $D:=\left\{z \in \mathbf{R}^{N}: z_{N}>0\right\}$. Then $w_{\epsilon} \rightarrow w$ in $C_{l o c}^{2}(D)$ as $\epsilon \rightarrow 0$.

Proof. Since $\left\|w_{\epsilon}\right\|_{\infty}=\left\|u_{\epsilon}\right\|_{\infty}<u_{0}$, by a standard regularity argument (cf. [3]), we see that $\left\{w_{\epsilon}\right\}$ is a precompact in $C_{l o c}^{2}$. Therefore, by choosing a subsequence if necessary, there exists $W \in C^{2}(D)$ such that $w_{\epsilon} \rightarrow W$ as $\epsilon \rightarrow 0$ in $C_{l o c}^{2}(D)$. Then we see from (2.4)-(2.9) that $W$ satisfies

$$
\begin{aligned}
-\Delta W & =f(W) \text { in } D, \\
W & >0 \text { in } D, \\
W & =0 \text { on } \partial D .
\end{aligned}
$$

It should be mentioned that (2.16) follows from 3. Now our aim is to show that $W=w$. To do this, we apply [3, Proposition 2.5]. That is, if we show

$$
\lim _{z_{N} \rightarrow \infty} W\left(\left(z^{\prime}, z_{N}\right)\right)=u_{0}
$$

uniformly for $z^{\prime} \in \mathbf{R}^{N-1}$, then we obtain $W=w$. Let an arbitrary $\eta \in \mathbf{R}^{N-1}$ be fixed. Furthermore, let an arbitrary $z_{N}>2 u_{0} / C_{1}$ be fixed, where $C_{1}>0$ is a constant defined in (2.19) below. Then there exists $0<\epsilon_{0} \ll 1$ such that $\left(\eta, z_{N}\right) \in B_{b / \epsilon}^{+}$for $0<\epsilon<\epsilon_{0}$. So $w_{\epsilon}\left(\left(\eta, z_{N}\right)\right)$ is well defined for $0<\epsilon<\epsilon_{0}$. Let an arbitrary $0<\tau<u_{0}$ be fixed. By [3 Lemma 3.1], we see that for all $x \in \Omega$,

$$
u_{\epsilon}(x)>\min \left\{C_{1} \operatorname{dist}(x, \partial \Omega) / \epsilon, \tau\right\},
$$

where $C_{1}>0$ is a constant. Since $\nabla \psi(0)=0$ by $(\psi 2)$, we see that $D \Phi(0)=I$, the identity map. Then by Taylor expansion, for $|z| \ll 1$, we have $\Phi(z)=\Phi(0)+$ $D \Phi(0) z+o(|z|)$. Therefore, since $\Phi(0)=0$,

$$
\Phi\left(\epsilon\left(\eta, z_{N}\right)\right)=\epsilon\left(\eta, z_{N}\right)+o\left(\epsilon \sqrt{|\eta|^{2}+z_{N}^{2}}\right) .
$$

Let $Q=Q_{\Phi\left(\epsilon\left(\eta, z_{N}\right)\right)}$ be the nearest point on $\partial \Omega$ from $\Phi\left(\epsilon\left(\eta, z_{N}\right)\right)$. Then since $Q \rightarrow 0$ as $\epsilon \rightarrow 0$, we see that $n_{Q} \rightarrow(0, \cdots, 0,1)$ as $\epsilon \rightarrow 0$, where $n_{Q}$ is the unit inner normal 
to $\partial \Omega$ at $Q$. Therefore, if $0<\epsilon \ll 1$, then $\operatorname{dist}\left(\Phi\left(\epsilon\left(\eta, z_{N}\right)\right), \partial \Omega\right)=\epsilon(1+o(1)) z_{N}$. Then by (2.19), for $0<\epsilon \ll 1$, we obtain

$$
w_{\epsilon}\left(\left(\eta, z_{N}\right)\right)=u_{\epsilon}\left(\Phi\left(\epsilon\left(\eta, z_{N}\right)\right)\right)>\min \left\{C_{1}(1-o(1)) z_{N}, \tau\right\}>\tau,
$$

since $z_{N}>2 u_{0} / C_{1}\left(>u_{0} /\left(C_{1}(1-o(1))\right)\right)$. Now let $\epsilon \rightarrow 0$. Then we see that $W\left(\left(\eta, z_{N}\right)\right)>\tau$ for any $\eta \in \mathbf{R}^{N-1}$ and $z_{N}>2 u_{0} / C_{1}$. This implies (2.18). Then we see that, from any subsequence of $\left\{w_{\epsilon}\right\}$, we can choose a subsequence of $\left\{w_{\epsilon_{j}}\right\}$, which satisfies $\epsilon_{j} \rightarrow 0$ as $j \rightarrow \infty$ and $w_{\epsilon_{j}} \rightarrow w$ in $C_{l o c}^{2}(D)$ as $j \rightarrow \infty$. This implies that $w_{\epsilon} \rightarrow w$ in $C_{l o c}^{2}(D)$ as $\epsilon \rightarrow 0$. Thus the proof is complete.

Therefore, we find that the top term of $\left(\partial u_{\epsilon} / \partial \nu\right)(0)$ can be obtained from $w^{\prime}(0)$. That is, by Lemma 2.1 and (2.12), as $\epsilon \rightarrow 0$,

$$
-\epsilon \frac{\partial u_{\epsilon}}{\partial \nu}(0)=\frac{\partial w_{\epsilon}}{\partial z_{N}}(0) \rightarrow \sqrt{2 F\left(u_{0}\right)} .
$$

Next, for $z=\left(z^{\prime}, z_{N}\right) \in B_{b / \epsilon}^{+}$, we put

$$
\phi_{\epsilon}(z):=\frac{w_{\epsilon}(z)-w\left(z_{N}\right)}{\epsilon} .
$$

Then it follows from (2.4) that for $z \in B_{b / \epsilon}^{+}$,

$$
\begin{aligned}
& \sum_{i, j=1}^{N}\left(\delta_{i j}+2 \epsilon \psi_{i j} z_{N}+\alpha_{i j}(z)\right)\left(\frac{\partial^{2} w}{\partial z_{i} \partial z_{j}}+\epsilon \frac{\partial^{2} \phi_{\epsilon}}{\partial z_{i} \partial z_{j}}\right) \\
& +\epsilon \sum_{j=1}^{N} b_{j}(z)\left(\frac{\partial w}{\partial z_{j}}+\epsilon \frac{\partial \phi_{\epsilon}}{\partial z_{j}}\right)+f\left(w+\epsilon \phi_{\epsilon}\right)=0 .
\end{aligned}
$$

Therefore, by (2.5)-(2.9), for $z \in B_{b / \epsilon}^{+}$,

$$
\begin{aligned}
& \sum_{i, j=1}^{N}\left(\delta_{i j}+2 \epsilon \psi_{i j} z_{N}+\alpha_{i j}(z)\right) \frac{\partial^{2} \phi_{\epsilon}}{\partial z_{i} \partial z_{j}}+\frac{\alpha_{N N}(z)}{\epsilon} w^{\prime \prime}\left(z_{N}\right)+\epsilon \sum_{j=1}^{N-1} \beta_{j}(z) \frac{\partial \phi_{\epsilon}}{\partial z_{j}} \\
& +\left(-(N-1) H(0)+\beta_{N}(z)\right)\left(w^{\prime}\left(z_{N}\right)+\epsilon \frac{\partial \phi_{\epsilon}}{\partial z_{N}}\right)+\frac{f\left(w+\epsilon \phi_{\epsilon}\right)-f(w)}{\epsilon}=0 .
\end{aligned}
$$

Then it is expected that the second term should be derived from the derivative of $\phi_{\epsilon}$.

Lemma 2.2. Let an arbitrary compact set $K \subset \bar{D}$ be fixed. Then there exists a constant $C_{K}>0$ such that $\left\|\phi_{\epsilon}\right\|_{\infty, K}:=\sup _{z \in K}\left|\phi_{\epsilon}(z)\right| \leq C_{K}$ for any $0<\epsilon \ll 1$.

Proof. Without loss of generality, we may assume that $K=\bar{B}_{R}^{+}$. Assume that there exists a subsequence of $\left\{\phi_{\epsilon}\right\}$, denoted by $\left\{\phi_{\epsilon}\right\}$ again, such that $\left\|\phi_{\epsilon}\right\|_{\infty, K} \rightarrow \infty$ as $\epsilon \rightarrow 0$. Since $\Omega$ satisfies the uniform interior sphere condition, there exists a constant $0<\xi_{1} \ll 1$ such that the ball $B_{\xi_{1}}\left(Q-\xi_{1} \nu_{Q}\right)$ is tangent to $\partial \Omega$ at $Q \in \partial \Omega$ and satisfies $B_{\xi_{1}}\left(Q-\xi_{1} \nu_{Q}\right) \subset \Omega$ for all $Q \in \partial \Omega$, where $\nu_{Q}$ is an outward unit vector to $\Omega$ at $Q \in \partial \Omega$. Moreover, since $\Omega$ satisfies the uniform exterior sphere condition and is bounded, there exist constants $0<\xi_{2} \ll 1$ and $\xi_{3} \gg 1$ such that the annulus $\Omega \subset A_{Q, \xi_{2}, \xi_{3}}:=\left\{x \in \mathbf{R}^{N}: \xi_{2}<\left|x-\left(Q+\xi_{2} \nu_{Q}\right)\right|<\xi_{3}\right\}$ and inner ball $B_{\xi_{2}}\left(Q+\xi_{2} \nu_{Q}\right)$ is tangent to $\partial \Omega$ at $Q$ for all $Q \in \partial \Omega$. Let $\phi_{\epsilon, Q}$ and $\psi_{\epsilon, Q}$ be the unique solutions 
to $(1.1)-(1.3)$, in which $\Omega$ is replaced by $B_{\xi_{1}}\left(Q-\xi_{1} \nu_{Q}\right)$ and $A_{Q, \xi_{2}, \xi_{3}}$, respectively. Then it follows from maximum principle that

$$
\phi_{\epsilon, Q}<u_{\epsilon}<\psi_{\epsilon, Q}
$$

This implies that (we write $\nu=\nu_{Q}$ for short)

$$
\frac{\partial \psi_{\epsilon, Q}}{\partial \nu}(Q)<\frac{\partial u_{\epsilon}}{\partial \nu}(Q)<\frac{\partial \phi_{\epsilon, Q}}{\partial \nu}(Q) .
$$

Moreover, we know from [12, Theorems 1 and 2] (cf. 11] for the case $f(u)=u-u^{p}$ ) that as $\epsilon \rightarrow 0$,

$$
\begin{aligned}
& \frac{\partial \phi_{\epsilon, Q}}{\partial \nu}(Q)=-\sqrt{2 F\left(u_{0}\right)} \epsilon^{-1}+\frac{(N-1) C_{0}}{\sqrt{2 F\left(u_{0}\right)} \xi_{1}}+O(\epsilon), \\
& \frac{\partial \psi_{\epsilon, Q}}{\partial \nu}(Q)=-\sqrt{2 F\left(u_{0}\right)} \epsilon^{-1}-\frac{(N-1) C_{0}}{\sqrt{2 F\left(u_{0}\right)} \xi_{2}}+O(\epsilon) .
\end{aligned}
$$

Therefore, we see that for any $Q \in \partial \Omega$ and $0<\epsilon \ll 1$,

$$
\frac{\partial u_{\epsilon}}{\partial \nu}(Q)=-\sqrt{2 F\left(u_{0}\right)} \epsilon^{-1}+O(1) \text {. }
$$

Then by this, for $\left(z^{\prime}, 0\right) \in \partial \bar{B}_{R}^{+}$, we obtain

$$
\begin{aligned}
\frac{\partial w_{\epsilon}}{\partial z_{N}}\left(z^{\prime}, 0\right) & =\epsilon\left(-\nabla \psi\left(\Phi\left(\left(\epsilon z^{\prime}, 0\right)\right)\right), 1\right) \cdot \nabla u_{\epsilon}\left(\Phi\left(\left(\epsilon z^{\prime}, 0\right)\right)\right) \\
& =-\epsilon \frac{\partial u_{\epsilon}}{\partial \nu}\left(\left(\epsilon z^{\prime}, \psi\left(\epsilon z^{\prime}\right)\right)\right) \\
& =\sqrt{2 F\left(u_{0}\right)}+O(\epsilon) .
\end{aligned}
$$

Therefore, by (2.12), (2.23) and (2.27), for $0<\epsilon \ll 1$,

$$
\frac{\partial \phi_{\epsilon}}{\partial z_{N}}\left(z^{\prime}, 0\right)=\frac{\left(\sqrt{2 F\left(u_{0}\right)}+O(\epsilon)\right)-\sqrt{2 F\left(u_{0}\right)}}{\epsilon}=O(1) .
$$

Now we put $\xi_{\epsilon}:=\phi_{\epsilon} /\left\|\phi_{\epsilon}\right\|_{\infty, K}$. Then clearly, $\left\|\xi_{\epsilon}\right\|_{\infty, K}=1$ and by (2.5)-(2.9) and (2.25), we can choose a subsequence of $\left\{\xi_{\epsilon}\right\}$ such that $\xi_{\epsilon} \rightarrow \xi$ in $C^{2}(K)$ as $\epsilon \rightarrow 0$. Moreover, we see from (2.25) and (2.28) that

$$
\begin{aligned}
\Delta \xi+f^{\prime}(w) \xi & =0 \text { in } K, \\
\xi & =0 \text { on } \partial K \\
\frac{\partial \xi}{\partial z_{N}} & =0 \text { on } \partial K .
\end{aligned}
$$

This implies that there exists $B_{\delta}^{+} \subset K$ such that $\xi \equiv 0$ in $B_{\delta}^{+}$. This along with the unique continuation theorem of second order elliptic equation, $\xi \equiv 0$ in $K$. This is a contradiction. Thus the proof is complete.

By Lemma 2.2 and (2.25), we see that by choosing a subsequence of $\left\{\phi_{\epsilon}\right\}$ if necessary, there exists $\phi \in C^{2}(D)$ such that as $\epsilon \rightarrow 0$,

$$
\phi_{\epsilon} \rightarrow \phi \quad \text { in } C_{l o c}^{2}(D),
$$

which satisfies

$$
\begin{aligned}
\Delta \phi+f^{\prime}(w) \phi & =(N-1) H(0) w^{\prime}\left(z_{N}\right) \text { in } D, \\
\phi & =0 \text { on } \partial D .
\end{aligned}
$$


We note that $\phi$ depends on the subsequence of $\{\epsilon\}$ at this stage. However, we see later that $\phi$ is uniquely determined.

Lemma 2.3. $\phi\left(z^{\prime}, z_{N}\right)=\phi\left(z_{N}\right)$ for $z \in D$.

Proof. Let an arbitrary compact subset $K \subset \partial D$ be fixed. We show that by choosing a subsequence of $\{\epsilon\}$ if necessary, there exists a constant $C_{2}>0$ such that for any $z^{\prime} \in K$, as $\epsilon \rightarrow 0$,

$$
\frac{\partial \phi_{\epsilon}}{\partial z_{N}}\left(z^{\prime}, 0\right) \rightarrow C_{2}
$$

We note that

$$
\begin{aligned}
\frac{\partial \phi_{\epsilon}}{\partial z_{N}}\left(z^{\prime}, 0\right) & =\left[-\frac{\partial u_{\epsilon}}{\partial \nu}\left(\epsilon z^{\prime}, \psi\left(\epsilon z^{\prime}\right)\right)+\frac{\partial u_{\epsilon}}{\partial \nu}(0,0)\right]+\left[-\frac{\partial u_{\epsilon}}{\partial \nu}(0,0)-\sqrt{2 F\left(u_{0}\right)} \epsilon^{-1}\right] \\
& :=I_{\epsilon}\left(z^{\prime}\right)+I I_{\epsilon} .
\end{aligned}
$$

We know from (2.26) that $\left|I I_{\epsilon}\right|$ is bounded. Therefore, by choosing a subsequence if necessary, we may assume that $I I_{\epsilon} \rightarrow C_{2}$ as $\epsilon \rightarrow 0$. We know that

$$
\begin{aligned}
I_{\epsilon}\left(z^{\prime}\right)= & -\left(\nabla \psi\left(\epsilon z^{\prime}\right),-1\right) \cdot\left(\frac{\partial u_{\epsilon}}{\partial x_{1}}\left(\epsilon z^{\prime}, \psi\left(\epsilon z^{\prime}\right)\right), \cdots, \frac{\partial u_{\epsilon}}{\partial x_{N}}\left(\epsilon z^{\prime}, \psi\left(\epsilon z^{\prime}\right)\right)\right) \\
& +(0,-1) \cdot\left(\frac{\partial u_{\epsilon}}{\partial x_{1}}(0,0), \cdots, \frac{\partial u_{\epsilon}}{\partial x_{N}}(0,0)\right) .
\end{aligned}
$$

Now we consider the cubes $Q_{1} \subset \Omega, \cdots, Q_{L} \subset \Omega$ satisfying the following properties:

(q.1) The length of the edge of $Q_{1}$ is $\epsilon^{k_{0}}$, where $k_{0}>0$ will be specified later. $Q_{j}(j=2, \cdots, L)$ is congruent to $Q_{1}$.

(q.2) A vertex $q_{j}$ of $Q_{j}(j=1, \cdots, L)$ is on $\partial \Omega$. In particular, a vertex $q_{1}$ of $Q_{1}$ coincides with the origin and a vertex $q_{L}$ of $Q_{L}$ coincides with $\left(\epsilon z^{\prime}, \psi\left(\epsilon z^{\prime}\right)\right)$. Moreover, there exists a constant $C>0$ such that $C^{-1} \epsilon^{k_{0}} \leq\left|q_{j+1}-q_{j}\right| \leq C \epsilon^{k_{0}}$ for $j=1, \cdots, L-1$.

(q.3) There exist $y_{j} \in Q_{j} \cap Q_{j+1}(j=1, \cdots, L-1)$ and a constant $C>0$ such that for $j=1, \cdots, L-1$,

$$
C^{-1} \epsilon^{k_{0}} \leq\left|q_{j+1}-y_{j}\right| \leq C \epsilon^{k_{0}}, C^{-1} \epsilon^{k_{0}} \leq\left|q_{j}-y_{j}\right| \leq C \epsilon^{k_{0}} .
$$

Then by (q.2), we see that there exists a constant $C>0$ such that $L \leq C \epsilon^{1-k_{0}}$. Moreover, we know from [1, p. 108] that for $p>N, t, s \in \bar{Q}_{j}(j=1, \cdots, L)$ and $m=1, \cdots, N$,

$$
\left|\frac{\partial u_{\epsilon}}{\partial x_{m}}(t)-\frac{\partial u_{\epsilon}}{\partial x_{m}}(s)\right| \leq C_{3}|t-s|^{1-N / p}\left\|u_{\epsilon}\right\|_{2, p, Q_{j}} .
$$

We know from [8] that for any $p>1$ and $0<\epsilon \ll 1$,

$$
\begin{aligned}
\left\|u_{\epsilon}\right\|_{2, p, \Omega} & \leq C_{4}\left(\frac{1}{\epsilon^{2}}\left\|f\left(u_{\epsilon}\right)\right\|_{0, p}+\left\|u_{\epsilon}\right\|_{0, p}\right) \\
& \leq C_{4}|\Omega|^{1 / p}\left(\max _{0 \leq s \leq u_{0}} f(s) \epsilon^{-2}+u_{0}\right) \\
& \leq 2 C_{4}|\Omega|^{1 / p} \epsilon^{-2} .
\end{aligned}
$$


By definition,

$$
\begin{aligned}
\left\|u_{\epsilon}\right\|_{2, p, Q_{j}}^{p}=\int_{Q_{j}}\left|u_{\epsilon}\right|^{p} d x+\sum_{m=1}^{N} \int_{Q_{j}}\left|\frac{\partial u_{\epsilon}}{\partial x_{m}}\right|^{p} d x \\
+\sum_{m, k=1}^{N} \int_{Q_{j}}\left|\frac{\partial^{2} u_{\epsilon}}{\partial x_{k} \partial x_{m}}\right|^{p} d x .
\end{aligned}
$$

Let $\xi=\left|u_{\epsilon}\right|,\left|\partial u_{\epsilon} / \partial x_{m}\right|$, or $\left|\partial^{2} u_{\epsilon} / \partial x_{m} \partial x_{k}\right|$. Let $\chi_{Q_{j}}$ be a characteristic function of $Q_{j}$. Then for $\alpha, \beta>1$ satisfying $1 / \alpha+1 / \beta=1$, by Hölder's inequality,

$$
\int_{Q_{j}} \xi^{p} d x=\int_{\Omega} \xi^{p} \chi_{Q_{j}} d x \leq\|\xi\|_{0, p \alpha}^{p}\left|Q_{j}\right|^{1 / \beta} \leq C_{5} \epsilon^{k_{0} N / \beta}\|\xi\|_{0, p \alpha, \Omega}^{p}
$$

Then we obtain

$$
\left\|u_{\epsilon}\right\|_{2, p, Q_{j}} \leq C_{6} \epsilon^{k_{0} N /(p \beta)}\left\|u_{\epsilon}\right\|_{2, p \alpha, \Omega} .
$$

Let $\bar{\alpha}, \bar{\beta}>1$ satisfy $1 / \bar{\alpha}+1 / \bar{\beta}=1$. Then by the same argument as above, we obtain

$$
\left\|u_{\epsilon}\right\|_{2, p \alpha, Q_{j}} \leq C_{7} \epsilon^{k_{0} N /(p \bar{\beta})}\left\|u_{\epsilon}\right\|_{2, p \alpha \bar{\alpha}, \Omega} .
$$

Since (2.35), in which $p$ is replaced by $p \alpha \bar{\alpha}$ is also valid, by (2.36), (2.39) and (2.40), we obtain

$$
\begin{aligned}
\left\|u_{\epsilon}\right\|_{2, p, Q_{j}} & \leq C_{8} \epsilon^{k_{0} N /(p \beta)+k_{0} N /(p \bar{\beta})}\left\|u_{\epsilon}\right\|_{2, p \alpha \bar{\alpha}, \Omega} \\
& \leq C_{9} \epsilon^{k_{0} N /(p \beta)+k_{0} N /(p \bar{\beta})-2} .
\end{aligned}
$$

Then by (q.3), (2.35) and (2.41)

$$
\begin{aligned}
\mid \frac{\partial u_{\epsilon}}{\partial x_{m}} & \left(\epsilon z^{\prime}, \psi\left(\epsilon z^{\prime}\right)\right)-\frac{\partial u_{\epsilon}}{\partial x_{m}}(0,0) \mid \\
& =\left|\frac{\partial u_{\epsilon}}{\partial x_{m}}\left(q_{L}\right)-\frac{\partial u_{\epsilon}}{\partial x_{m}}\left(q_{1}\right)\right| \\
& \leq \sum_{j=1}^{L-1}\left|\frac{\partial u_{\epsilon}}{\partial x_{m}}\left(q_{j+1}\right)-\frac{\partial u_{\epsilon}}{\partial x_{m}}\left(q_{j}\right)\right| \\
& \leq \sum_{j=1}^{L-1}\left(\left|\frac{\partial u_{\epsilon}}{\partial x_{m}}\left(q_{j+1}\right)-\frac{\partial u_{\epsilon}}{\partial x_{m}}\left(y_{j}\right)\right|+\left|\frac{\partial u_{\epsilon}}{\partial x_{m}}\left(y_{j}\right)-\frac{\partial u_{\epsilon}}{\partial x_{m}}\left(q_{j}\right)\right|\right) \\
& \leq C_{10} \epsilon^{\left(k_{0} N / p\right)(1 / \beta+1 / \bar{\beta}-1)-1} .
\end{aligned}
$$

Choose $k_{0} \gg 1$ and $0<\delta \ll 1$ and put $p=N+\delta, \beta=\bar{\beta}=1+\delta$ so that $\left(k_{0} N / p\right)(1 / \beta+1 / \bar{\beta}-1)-1>0$. Then by $(\psi 2)$ and $(2.42)$, we see that $I_{\epsilon}\left(z^{\prime}\right) \rightarrow 0$ uniformly on $K$ as $\epsilon \rightarrow 0$. Therefore, by this and (2.33), we obtain (2.32), and (2.32) implies that $\left(\partial \phi / \partial z_{N}\right)\left(z^{\prime}, 0\right)=C_{2}$ on $K \cap \partial D$. Now let $\varphi$ be a unique solution to the ODE

$$
\begin{aligned}
& \varphi^{\prime \prime}(t)+f^{\prime}(w(t)) \varphi(t)=(N-1) H(0) w^{\prime}(t), \quad t>0, \\
& \varphi(0)=0, \\
& \varphi^{\prime}(0)=C_{2} .
\end{aligned}
$$


Now put $\eta(z):=\phi(z)-\varphi\left(z_{N}\right)$. Then by $(2.30),(2.31)$ and $(2.43)-(2.45), \eta$ satisfies

$$
\begin{aligned}
\Delta \eta+f^{\prime}(w) \eta & =0 \text { in } K, \\
\eta & =\frac{\partial \eta}{\partial z_{N}}=0 \quad \text { on } K \cap \partial D .
\end{aligned}
$$

This implies that $\eta \equiv 0$ on $K$. Then unique continuation theorem of elliptic equation implies $\phi(z)=\varphi\left(z_{N}\right)$. Thus the proof is complete.

Note that $\phi$ still depends on the choice of the subsequence of $\{\epsilon\}$ at this stage. To show that $\phi$ is independent of the choice of the subsequence of $\{\epsilon\}$, we shall prove that $\phi^{\prime}(0)$ is independent of choice of the subsequence of $\{\epsilon\}$. To do this, we need the following lemma.

Lemma 2.4. $\|\phi\|_{\infty} \leq C$.

Proof. We put $g_{\epsilon}\left(z_{N}\right):=\phi_{\epsilon}\left(0, \cdots, 0, z_{N}\right)$. We show that for $0<\epsilon \ll 1$,

$$
\left\|g_{\epsilon}\right\|_{L^{\infty}(R)} \leq C \text {. }
$$

Then by letting $\epsilon \rightarrow 0$, we obtain our assertion. The proof is divided into four steps.

Step 1. Let $B_{1}:=\left\{x_{1}^{2}+\cdots+\left(x_{N}-r_{1}\right)^{2}<r_{1}^{2}\right\} \subset M \subset \Omega$, where $r_{1}>0$ $\left(r_{1}<b\right)$ is a small constant and $M$ is defined in $(\psi 3)$ and $(\psi 4)$. Furthermore, let $U_{1, \epsilon}$ be a unique solution to (1.1)-(1.3) in which $\Omega$ is replaced by $B_{1}$. Since $U_{1, \epsilon}$ is a subsolution to (1.1)-(1.3), we see that for $0<\epsilon \ll 1$,

$$
U_{1, \epsilon}(x) \leq u_{\epsilon}(x), \quad x \in B_{1} .
$$

As for the subsolution, we refer to [3, Appendix]. Now for $z_{N} \in B_{b / \epsilon}^{+}$, we put

$$
\zeta_{\epsilon}\left(z_{N}\right):=\frac{U_{1, \epsilon}\left(\Phi\left(\epsilon\left(0, \cdots, 0, z_{N}\right)\right)-w\left(z_{N}\right)\right.}{\epsilon}=\frac{U_{1, \epsilon}\left(\epsilon z_{N}\right)-w\left(z_{N}\right)}{\epsilon} .
$$

By (2.23) and (2.47),

$$
g_{\epsilon}\left(z_{N}\right) \geq \zeta_{\epsilon}\left(z_{N}\right) .
$$

Step 2. (a) We show that $\left\|\zeta_{\epsilon}\right\|_{\infty} \leq C$ for $0<\epsilon \ll 1$. Let $\tilde{B}_{0}:=\left\{|x|<r_{1}\right\}$ and $V_{1, \epsilon}=V_{1, \epsilon}(r) \quad(r=|x|)$ be a unique solution to (1.1)-(1.3), in which $\Omega$ is replaced by $\tilde{B}_{0}$. Then $V_{1, \epsilon}$ satisfies

$$
-\epsilon^{2}\left(V_{1, \epsilon}^{\prime \prime}(r)+\frac{N-1}{r} V_{1, \epsilon}^{\prime}(r)\right)=f\left(V_{1, \epsilon}(r)\right), \quad 0<r<r_{1} .
$$

Put $s=r_{1}-r$. Then clearly, $U_{1, \epsilon}(s)=V_{1, \epsilon}(r)$ and $U_{1, \epsilon}$ satisfies

$$
-\epsilon^{2}\left\{U_{1, \epsilon}^{\prime \prime}(s)-\frac{N-1}{r_{1}-s} U_{1, \epsilon}^{\prime}(s)\right\}=f\left(U_{1, \epsilon}(s)\right), \quad 0<s<r_{1} .
$$

By this, (2.10), (2.48) and mean value theorem, we obtain

$$
-\zeta_{\epsilon}^{\prime \prime}\left(z_{N}\right)+\frac{N-1}{r_{1}-\epsilon z_{N}}\left(w^{\prime}\left(z_{N}\right)+\epsilon \zeta_{\epsilon}^{\prime}\left(z_{N}\right)\right)=f^{\prime}\left(w\left(z_{N}\right)+\theta_{\epsilon} \epsilon \zeta_{\epsilon}\left(z_{N}\right)\right) \zeta_{\epsilon}\left(z_{N}\right),
$$

where $0<\theta_{\epsilon}:=\theta_{\epsilon}\left(z_{N}\right)<1$. Let

$$
\bar{\zeta}_{\epsilon}(z):=\frac{U_{1, \epsilon}(\Phi(\epsilon z))-w\left(z_{N}\right)}{\epsilon}
$$


Then by Lemma 2.2 for the case $\Omega=B_{1}$, we see that $\left\|\bar{\zeta}_{\epsilon}\right\|_{\infty} \leq C_{K}$ for any compact subset $K \subset \bar{D}$ and $0<\epsilon \ll 1$. Since $\zeta_{\epsilon}\left(z_{N}\right)=\bar{\zeta}_{\epsilon}\left(0, z_{N}\right)$, we see that $\left|\zeta_{\epsilon}\left(z_{N}\right)\right| \leq C_{l}$ for any $0 \leq z_{N} \leq l$.

(b) Now assume that there exists a sequence $\left\{z_{N, \epsilon}\right\}\left(z_{N, \epsilon} \in B_{r_{1} / \epsilon}^{+}\right)$such that $z_{N, \epsilon} \rightarrow \infty$ and $\left\|\zeta_{\epsilon}\right\|_{\infty}=\left|\zeta_{\epsilon}\left(z_{N, \epsilon}\right)\right| \rightarrow \infty$ as $\epsilon \rightarrow 0$. Let $0<\delta_{0}<r_{1}$ be a fixed constant. We know (see Appendix) that for $0<s<r_{1}$ and $0<\epsilon \ll 1$,

$$
u_{0}-C \exp (-C s / \epsilon) \leq U_{1, \epsilon}(s) \leq u_{0} .
$$

Moreover, we know that for $t \gg 1$,

$$
w(t)=u_{0}-O\left(e^{-C_{10} t}\right) .
$$

By this and (2.52), we see that if there exists a constant $C_{11}>0$ such that $\epsilon z_{N, \epsilon} \geq$ $C_{11}$, then as $\epsilon \rightarrow 0$,

$$
\zeta_{\epsilon}\left(z_{N, \epsilon}\right)=O\left(\frac{e^{-C_{12} / \epsilon}}{\epsilon}\right) \rightarrow 0 .
$$

Therefore, $\epsilon z_{N, \epsilon} \rightarrow 0$ as $\epsilon \rightarrow 0$.

Step 3. Under the assumption of Step 2 (b), we put $\eta_{\epsilon}(t):=\zeta_{\epsilon}\left(z_{N}-z_{N, \epsilon}\right) /\left\|\zeta_{\epsilon}\right\|_{\infty}$, where $t=z_{N}-z_{N, \epsilon}$ and consider (2.51) in the interval $J:=\left(-\delta z_{N, \epsilon}, \delta z_{N, \epsilon}\right)$, where $\delta>0$ is a small constant. Then by $(2.51)$, for $t \in J$, we have

$$
\begin{gathered}
-\eta_{\epsilon}^{\prime \prime}(t)+\frac{N-1}{r_{1}-\epsilon\left(t+z_{N, \epsilon}\right)}\left\{\frac{w^{\prime}\left(t+z_{N, \epsilon}\right)}{\left\|\zeta_{\epsilon}\left(z_{N}\right)\right\|_{\infty}}+\epsilon \eta_{\epsilon}^{\prime}(t)\right\} \\
=f^{\prime}\left(W(t)+\theta_{\epsilon} \epsilon \zeta_{\epsilon}\left(t+z_{N, \epsilon}\right)\right) \eta_{\epsilon}(t),
\end{gathered}
$$

where $W(t)=w\left(z_{N}\right)$. We show that

$$
\epsilon \zeta_{\epsilon}\left(t+z_{N, \epsilon}\right)=\epsilon \zeta_{\epsilon}\left(z_{N}\right)=U_{1, \epsilon}\left(\epsilon z_{N}\right)-w\left(z_{N}\right) \rightarrow 0
$$

uniformly in $J$ as $\epsilon \rightarrow 0$. Indeed, if $t \in J$, then clearly, we have

$$
(1-\delta) z_{N, \epsilon} \leq z_{N}=t+z_{N, \epsilon} \leq(1+\delta) z_{N, \epsilon} .
$$

This implies that if $t \in J$, then $z_{N} \rightarrow \infty$ as $\epsilon \rightarrow 0$ uniformly on $J$. Then by (2.52) and (2.53), as $\epsilon \rightarrow 0$,

$$
\begin{aligned}
\left|\epsilon \zeta_{\epsilon}\left(t+z_{N, \epsilon}\right)\right| & =\left|\epsilon \zeta_{\epsilon}\left(\epsilon z_{N}\right)\right| \leq\left|u_{0}-U_{1, \epsilon}\left(\epsilon z_{N}\right)\right|+\left|u_{0}-w\left(z_{N}\right)\right| \\
& =O\left(\exp \left(-C z_{N}\right)\right)+O\left(\exp \left(-C z_{N}\right)\right) \\
& \rightarrow 0
\end{aligned}
$$

Then by the limiting procedure, we obtain that there exists $\eta \in C^{2}(\mathbf{R})$ such that

$$
\begin{aligned}
\eta^{\prime \prime}(t) & =-f^{\prime}\left(u_{0}\right) \eta(t), \quad t \in \mathbf{R} \\
\|\eta\|_{\infty} & =\eta(0)=1 .
\end{aligned}
$$

By solving the first equation, we obtain $\eta(t)=c_{1} e^{-\sqrt{-f^{\prime}\left(u_{0}\right)} t}+c_{2} e^{\sqrt{-f^{\prime}\left(u_{0}\right)} t}$ for $t \in \mathbf{R}$. However, this is impossible, since $\|\eta\|_{\infty}=1$. Therefore, $\zeta_{\epsilon}\left(z_{N}\right)$ is bounded.

Step 4. Finally, let $A_{1}:=\left\{a^{2}<x_{1}^{2}+\cdots+\left(x_{N}+a\right)^{2}<R^{2}\right\}$ which satisfies $\Omega \subset A_{1}$, where $a>0$ is a small constant and $R>0$ is a large constant. Furthermore, let $U_{2, \epsilon}$ be a unique solution to (1.1)-(1.3) in which $\Omega$ is replaced by $A_{1}$. Since $U_{2, \epsilon}$ is a supersolution to (1.1)-(1.3), we see that for $0<\epsilon \ll 1$,

$$
U_{2, \epsilon}(x) \geq u_{\epsilon}(x), \quad x \in \Omega .
$$


Now for $z_{N} \in B_{b / \epsilon}^{+}$, we put

$$
\zeta_{2, \epsilon}\left(z_{N}\right):=\frac{U_{2, \epsilon}\left(\Phi\left(\epsilon\left(0, \cdots, 0, z_{N}\right)\right)-w\left(z_{N}\right)\right.}{\epsilon} .
$$

Then by (2.23) and (2.60),

$$
g_{\epsilon}\left(z_{N}\right) \leq \zeta_{2, \epsilon}\left(z_{N}\right)
$$

Then by the same argument as that just above, we also obtain that $\zeta_{2, \epsilon}\left(z_{N}\right)$ is bounded. Hence, by (2.49) and (2.62), we obtain our assertion.

By Lemma 2.4 and (2.43), we see that $\left\|\phi^{\prime}\right\|_{\infty} \leq C$.

Lemma 2.5. $\phi^{\prime}(0)=-(N-1) H(0) C_{0} / \sqrt{2 F\left(u_{0}\right)}$.

Proof. Multiply (2.43) by $w^{\prime}$ and integrate it over $(0, R)$. Then

$$
\int_{0}^{R} \phi^{\prime \prime}(t) w^{\prime}(t) d t+\int_{0}^{R} f^{\prime}(w(t)) w^{\prime}(t) \phi(t) d t=(N-1) H(0) \int_{0}^{R} w^{\prime}(t)^{2} d t .
$$

Then integration by parts yields

$$
\phi^{\prime}(R) w^{\prime}(R)-\phi^{\prime}(0) w^{\prime}(0)+f(w(R)) \phi(R)=(N-1) H(0) \int_{0}^{R} w^{\prime}(t)^{2} d t .
$$

Let $R \rightarrow \infty$. Then by (2.12), (2.14) and (f.2), we obtain

$$
\phi^{\prime}(0)=-\frac{(N-1) H(0)}{\sqrt{2 F\left(u_{0}\right)}} \int_{0}^{\infty} w^{\prime}(t)^{2} d t .
$$

So the last task for us is to calculate $\int_{0}^{\infty} w^{\prime}(t)^{2} d t$. Multiply (2.10) by $w^{\prime}$. Then we have

$$
\frac{d}{d t}\left(\frac{1}{2} w^{\prime}(t)^{2}+F(w(t))\right) \equiv 0 .
$$

This implies that $w^{\prime}(t)^{2} / 2+F(w(t)) \equiv$ constant for $t \geq 0$. Then by (2.12) and putting $t=0$, for $t \geq 0$, we obtain

$$
\frac{1}{2} w^{\prime}(t)^{2}+F(w(t))=\frac{1}{2} w^{\prime}(0)^{2}=F\left(u_{0}\right) .
$$

By (2.13), for $t \geq 0$, we have

$$
w^{\prime}(t)=\sqrt{2\left(F\left(u_{0}\right)-F(w(t))\right.} .
$$

By this and (2.14)

$$
\begin{aligned}
\int_{0}^{\infty} w^{\prime}(t)^{2} d t & =\int_{0}^{\infty} \sqrt{2\left(F\left(u_{0}\right)-F(w(t))\right.} w^{\prime}(t) d t \\
& =\int_{0}^{u_{0}} \sqrt{2\left(F\left(u_{0}\right)-F(s)\right.} d s=C_{0} .
\end{aligned}
$$

This along with (2.65) implies our assertion.

Since we have

$$
\begin{aligned}
-\epsilon \frac{\partial u_{\epsilon}}{\partial \nu}(0) & =\frac{\partial w_{\epsilon}}{\partial z_{N}}(0)=w^{\prime}(0)+\epsilon \frac{\partial \phi_{\epsilon}}{\partial z_{N}}(0) \\
& =\sqrt{2 F\left(u_{0}\right)}+\epsilon\left(\phi^{\prime}(0)+o(1)\right),
\end{aligned}
$$

this along with Lemma 2.5 implies Theorem 1.1. Thus we get Theorem 1.1. 


\section{Appendix}

In this section, we prove (2.52) for completeness. We consider the equation

$$
\begin{aligned}
& \epsilon^{2}\left(V_{1, \epsilon}^{\prime \prime}(r)+\frac{N-1}{r} V_{1, \epsilon}^{\prime}(r)\right)+f\left(V_{1, \epsilon}(r)\right)=0,0<r<r_{1}, \\
& V_{1, \epsilon}(r)>0, \quad 0<r<r_{1}, \\
& V_{1, \epsilon}^{\prime}(0)=V_{1, \epsilon}\left(r_{1}\right)=0 .
\end{aligned}
$$

It is known (cf. [5]) that by the change of independent variable

$$
v_{\epsilon}(t):=V_{1, \epsilon}(r), \quad t:=h(r)=\left\{\begin{array}{l}
\frac{1}{N-2}\left[\frac{1}{r_{1}^{N-2}}-\frac{1}{r^{N-2}}\right] \quad(N \geq 3), \\
\log \frac{r}{r_{1}}(N=2),
\end{array}\right.
$$

(3.1)-(3.3) is transformed into

$$
\begin{aligned}
-\epsilon^{2} v^{\prime \prime}(t) & =g(t) f(v(t)), \quad-\infty<t<0 \\
v(t) & >0,-\infty<t<0 \\
v(0) & =v^{\prime}(-\infty)=0
\end{aligned}
$$

and $v_{\epsilon}$ is a solution to $(3.5)-(3.7)$, where $g(t)=\left[h^{-1}(t)\right]^{2(N-1)}$. Let $a>0$ be fixed and consider here the auxiliary problem

$$
\begin{aligned}
-\epsilon^{2} W^{\prime \prime}(t) & =g(-a) f(W(t)),-a<t<0, \\
W(t) & >0,-a<t<0, \\
W(-a) & =W(0)=0 .
\end{aligned}
$$

This equation has a unique solution $W_{\epsilon}$ if $0<\epsilon \ll 1$. Then we know from [5] that

$$
W_{\epsilon}(t) \leq v_{\epsilon}(t),-a \leq t \leq 0 .
$$

Lemma 3.1. There exist constants $C_{2}, C_{3}>0$ such that for $-a<t<0$ and $0<\epsilon \ll 1$

$$
0<u_{0}-C_{2} \exp \left(C_{3} t / \epsilon\right) \leq W_{\epsilon}(t) .
$$

Proof. Multiply (3.8) by $W_{\epsilon}^{\prime}(t)$. Then for $-a \leq t \leq 0$, we obtain

$$
\left(\epsilon^{2} W_{\epsilon}^{\prime \prime}(t)+g(-a) f\left(W_{\epsilon}(t)\right)\right) W_{\epsilon}^{\prime}(t)=0 .
$$

This implies that

$$
\frac{d}{d t}\left\{\frac{1}{2} \epsilon^{2} W_{\epsilon}^{\prime}(t)^{2}+g(-a) F\left(W_{\epsilon}(t)\right)\right\} \equiv 0 .
$$

So, by putting $t=-a / 2$, we obtain

$$
\frac{1}{2} \epsilon^{2} W_{\epsilon}^{\prime}(t)^{2}+g(-a) F\left(W_{\epsilon}(t)\right)=g(-a) F\left(\left\|W_{\epsilon}\right\|_{\infty}\right) .
$$

Then since $W_{\epsilon}^{\prime}(t)<0$ for $-a / 2<t<0$, we have

$$
-W_{\epsilon}^{\prime}(t)=\frac{1}{\epsilon} \sqrt{g(-a)} \sqrt{2 F\left(\left\|W_{\epsilon}\right\|_{\infty}\right)-2 F\left(W_{\epsilon}(t)\right)} .
$$


Let an arbitrary $0<\delta \ll 1$ be fixed. Then for $-a / 2<-t_{1}<0$,

$$
\begin{aligned}
t_{1} & =\int_{-t_{1}}^{0} d t \\
& =\int_{-t_{1}}^{0} \frac{-\epsilon W_{\epsilon}^{\prime}(t)}{\sqrt{g(-a)} \sqrt{2 F\left(\left\|W_{\epsilon}\right\|_{\infty}\right)-2 F\left(W_{\epsilon}(t)\right)}} d t \\
& =\frac{\epsilon}{\sqrt{g(-a)}} \int_{0}^{W_{\epsilon}\left(-t_{1}\right)} \frac{1}{\sqrt{2 F\left(\left\|W_{\epsilon}\right\|_{\infty}\right)-2 F(s)}} d s \\
& =\frac{\epsilon}{\sqrt{g(-a)}} \int_{u_{0}-W_{\epsilon}\left(-t_{1}\right)}^{u_{0}} \frac{1}{\sqrt{2 F\left(\left\|W_{\epsilon}\right\|_{\infty}\right)-2 F\left(u_{0}-\eta\right)}} d \eta \\
& =\frac{\epsilon}{\sqrt{g(-a)}}\left[\int_{\delta}^{u_{0}}+\int_{u_{0}-W_{\epsilon}\left(-t_{1}\right)}^{\delta}\right] .
\end{aligned}
$$

Let $0<C_{4}<-f^{\prime}\left(u_{0}\right)$ be fixed. Then for $u_{0}-\left\|W_{\epsilon}\right\|_{\infty} \leq \eta \leq \delta$,

$$
m(\eta):=2 F\left(\left\|W_{\epsilon}\right\|_{\infty}\right)-2 F\left(u_{0}-\eta\right)-C_{4} \eta^{2}+C_{4}\left(u_{0}-\left\|W_{\epsilon}\right\|_{\infty}\right)^{2}>0 .
$$

Indeed, by (f.3) and Taylor expansion, for $u_{0}-\left\|W_{\epsilon}\right\|_{\infty} \leq \eta \leq \delta$, we have

$$
\begin{aligned}
m^{\prime}(\eta) & =2 f\left(u_{0}-\eta\right)-2 C_{4} \eta \\
& =2\left(-f^{\prime}\left(u_{0}\right)-C_{4}\right) \eta-O\left(\eta^{2}\right)>0 .
\end{aligned}
$$

Since $m\left(u_{0}-\left\|W_{\epsilon}\right\|_{\infty}\right)=0$, by (3.17), we obtain (3.16). Then by (3.16), we obtain

$$
\begin{array}{r}
t_{1} \leq \frac{\epsilon}{\sqrt{g(-a)}}\left[C_{\delta}+\int_{u_{0}-W_{\epsilon}\left(-t_{1}\right)}^{\delta} \frac{1}{\sqrt{C_{4} \eta^{2}-C_{4}\left(u_{0}-\left\|W_{\epsilon}\right\|_{\infty}\right)^{2}}} d t\right] \\
=\frac{\epsilon}{\sqrt{g(-a)}}\left[C_{\delta}+\frac{1}{\sqrt{C_{4}}} \log \left|\delta+\sqrt{\delta^{2}-\left(u_{0}-\left\|W_{\epsilon}\right\|_{\infty}\right)^{2}}\right|\right. \\
\left.-\frac{1}{\sqrt{C_{4}}} \log \left|\left(u_{0}-W_{\epsilon}\left(-t_{1}\right)\right)\right|\right] .
\end{array}
$$

This implies

$$
\log \left|u_{0}-W_{\epsilon}\left(-t_{1}\right)\right| \leq \bar{C}_{\delta}-\frac{\sqrt{C_{4} g(-a)}}{\epsilon} t_{1} .
$$

This yields (3.12).

By (3.4), let

$$
r_{0}:=\left\{\begin{array}{l}
\left(\frac{2 r_{1}^{N-2}}{2+a(N-2) r_{1}^{N-2}}\right)^{1 /(N-2)} \quad(N \geq 3), \\
r_{1} e^{-a / 2}(N=2) .
\end{array} \quad\right.
$$

Then by this, Lemma 3.1 and a direct calculation, for $r_{0} \leq r<r_{1}$, we obtain

$$
u_{0}-C_{3} \exp \left(-C_{4}\left(r_{1}-r\right) / \epsilon\right) \leq V_{1, \epsilon}(r)<u_{0} .
$$

Then since $V_{1, \epsilon}(r) \geq V_{1, \epsilon}\left(r_{0}\right)$ for $0 \leq r \leq r_{0}$, we obtain

$$
\begin{aligned}
u_{0}- & C_{3} \exp \left(-C_{4}\left(r_{1}-r_{0}\right)\left(r_{1}-r\right) /\left(r_{1} \epsilon\right)\right) \\
& \leq u_{0}-C_{3} \exp \left(-C_{4}\left(r_{1}-r_{0}\right) / \epsilon\right) \\
& \leq V_{1, \epsilon}\left(r_{0}\right) \leq V_{1, \epsilon}(r)<u_{0} .
\end{aligned}
$$


Then by (3.20) and (3.21), it is clear that (3.21) holds for $0 \leq r<r_{1}$ and $0<$ $\epsilon \ll 1$. Since $U_{1, \epsilon}(s)=V_{1, \epsilon}(r)$ and $s=r_{1}-r$, we obtain (2.52). Thus the proof is complete.

\section{ACKNOWLEDGMENT}

The author thanks the referee for his helpful comments.

\section{REFERENCES}

[1] R. A. Adams, Sobolev spaces, Academic Press, New York (1975).

[2] H. Berestycki, Le nombre de solutions de certains problèmes semi-linéares elliptiques, J. Functional Analysis 40 (1981), 1-29. MR 82k:35033

[3] P. Clément and G. Sweers, Existence and multiplicity results for a semilinear elliptic eigenvalue problems, Ann. Scuola Norm. Sup. Pisa (1987), 97-120. MR 89j:35053

[4] R. S. Cantrell and C. Cosner, Diffusive logistic equations with indefinite weights: population models in disrupted environments, Proc. Roy. Soc. Edinburgh Sect A 112 (1987), 293-318. MR 91b:92015

[5] J. M. Fraile, J. López-Gómez and J. C. Sabina de Lis, On the global structure of the set of positive solutions of some semilinear elliptic boundary value problems, J. Differential Equations 123 (1995), 180-212. MR 96j:35073

[6] D. G. Figueiredo, On the uniqueness of positive solutions of the Dirichlet problem $-\triangle u=$ $\lambda \sin u$, Pitman Res. Notes in Math. 122 (1985), 80-83.

[7] J. García-Melián and J. C. Sabina de Lis, Stationary profiles of degenerate problems when parameter is large, Diff. Int. Equations 13 (2000), 1201-1232. MR 2001h:35059

[8] D. Gilbarg and N. S. Trudinger, Elliptic partial differential equations of second order, Springer, New York (1983). MR 86c:35035

[9] F. A. Howes, Singularly perturbed semilinear elliptic boundary value problems, Comm. Partial Differential Equations 4 (1979), 1-39. MR 81i:35072

[10] W-M. Ni and I. Takagi, Locating the peaks of least-energy solutions to a semilinear Neumann problems, Duke Math. J. 70 (1993), 247-281. MR 94h:35072

[11] T. Shibata, Asymptotic formulas for boundary layers and eigencurves for nonlinear elliptic eigenvalue problems, Comm. Partial Differential Equations 28 (2003), 581-600.

[12] T. Shibata, Three-term asymptotics for the boundary layers of semilinear elliptic eigenvalue problems, to appear in Nonlinear Differential Equations and Applications.

The Division of Mathematical and Information Sciences, Faculty of Integrated Arts and Sciences, Hiroshima University, Higashi-Hiroshima, 739-8521, Japan

E-mail address: shibata@mis.hiroshima-u.ac.jp 